\title{
Die Suche nach dem Warum
}

\author{
WISSENSCHAFT ERKLÄRT: QUALITATIVE STUDIEN Wissenschaft bedeutet nicht \\ immer Zahlen und Statistiken. Während quantitative Studien sich mit dem „Wie \\ viel?“, „Wie oft?“ und „Wie effektiv?“ beschäftigen, zielen qualitative Studien auf \\ das zugrunde liegende Warum ab.
}

\section{B}

eschäftigt man sich mit Wissenschaft, so begegnet man schnell der Statistik und verliert sich in unendlichen Berechnungen. Die Fragen „Wie viele Patienten profitieren von der Forced-use-Therapie?“ und „Wie viele Physiotherapeuten studieren an einer Fachhochschule?" kann man konkret mit Zahlen beantworten. Das Warum dahinter ist schwerer zu erfassen und nur schwer mathematisch berechenbar.

Man benötigt dazu qualitative Studien. Durch offenes Fragen in Form eines Interviews lassen sich Hintergründe erfassen, Meinungen erörtern und Empfindungen analysieren. Forscher bringen Informationen aus nichtstandardisierten Daten in Zusammenhang. Die Frage, warum Physiotherapeuten immer häufiger ein Bachelorstudium an die Ausbildung anschließen, können sie oft nicht mit einem Satz beantworten. Forscher müssen die Studenten so offen wie möglich befragen und berichten lassen. Dann erfolgt die Deutung so neutral wie möglich. Potenzielle Fragen hierfür sind beispielsweise: „Welche Argumente gibt es für und gegen ein Physiotherapiestudium? Was interessiert Sie besonders am wissenschaftlichen Arbeiten? Was erwarten Sie von einem Aufbaustudium?" Diese Fragen lassen sich nicht messen und quantifizieren. Sie geben Auskunft über die subjektive Wahrnehmung der Befragten.

Neue Themen erforschen > Der wichtigste Aspekt qualitativer Studien ist die Erforschung neuer und unbekannter Themen. Wir lernen verstehen, wie Patienten oder Angehörige verschiedene Situationen erleben oder wie sie unterschiedliche Aspekte von Therapien empfinden. Als Methoden der qualitativen Forschung eignen sich unter anderem Fragebögen mit offenen Fragen, teilstrukturierte Interviews oder freie Berichte. Auch soge-

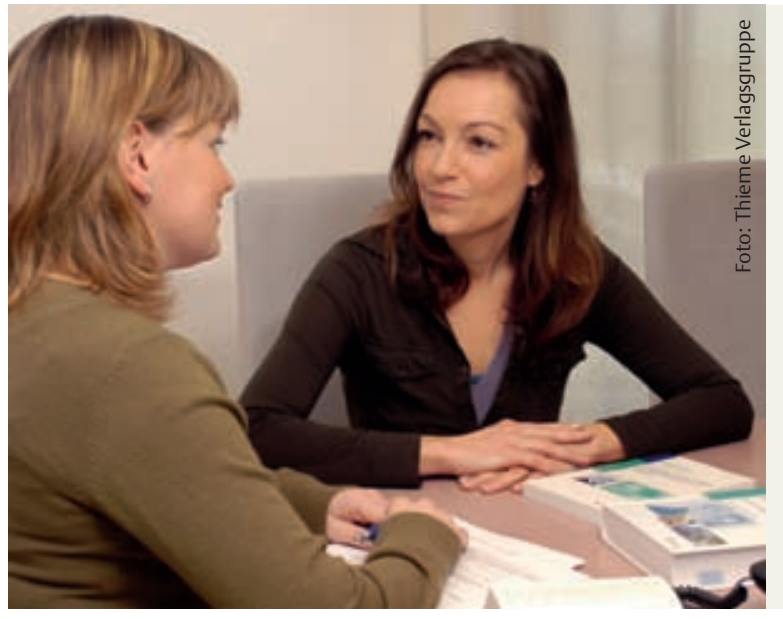

Ein teilstrukturiertes Interview ist eine gute Methode, um qualitativ zu forschen.

nannte Tiefeninterviews sind möglich. Es gibt mittlerweile eine ganze Bandbreite an Auswertungs- bzw. Analysemethoden für qualitative Studien, die sogar durch Statistikprogramme verarbeitet werden können.

Kontroverse Ergebnisse erklären > Ergebnisse aus qualitativen Studien sind wichtig und tragen auf unterschiedliche Art und Weise zur Forschung bei. Sie erörtern zum Beispiel, welche Kriterien für Patienten bei einer physiotherapeutischen Behandlung wichtig sind. Sie geben Erklärungen zu kontroversen Forschungsergebnissen aus quantitativen Studien oder analysieren die Sicht der Patienten auf eine Therapiemethode. Qualitative Forschung ist unerlässlich - genauso wie quantitative Forschung. Beide Arten der Forschung eignen sich für ganz bestimmte Fragestellungen und sind gleich wichtig. In der Wissenschaft werden sie gerne kombiniert: Wissenschaftler analysieren gefundene Variabeln aus qualitativen Studien in darauffolgenden quantitativen Studien weiter. Dies ist zum Beispiel der Fall, wenn man zuerst die Anforderungen von Patienten an eine Reha qualitativ erörtert und man dann in einer weiteren Studie quantitativ erfasst, ob diese Wünsche erfüllt werden und die Patienten tatsächlich zufrieden sind.

Kritik an qualitativer Forschung $>$ Kritiker werfen der qualitativen Forschung vor, subjektiv und willkürlich zu sein. Vor allem sei die Anzahl der Befragten und somit der Studienteilnehmer meist zu klein und deshalb nicht repräsentativ. Man hat jedoch in den letzten Jahren mehrere methodisch kontrollierte Verfahren wie die Grounded Theory, die objektive Hermeneutik und die qualitative Inhaltsanalyse entwickelt. Diese Verfahren enthalten Qualitätskriterien und Gütestandards, um die Nachvollziehbarkeit qualitativer Forschungsergebnisse zu verbessern.

Viele Gebiete der Physiotherapie müssen noch weiter erforscht werden. Um dies sinnvoll und ganzheitlich zu tun, sind sowohl quantitative als auch qualitative Studien wichtig. Optimalerweise sollten sich diese ergänzen. Oder warum sollte man eine Therapiemethode erforschen, von der man nicht weiß, ob die betrachtete Patientengruppe sie überhaupt benötigt?

Prof. Dr. Jan Mehrholz 